\title{
Reconstruction of Various Perinasal Defects Using Facial Artery Perforator-Based Nasolabial Island Flaps
}

\author{
Tae Ho Yoon, In Sik Yun, Dong Kyun Rha, Won Jai Lee \\ Department of Plastic and Reconstructive Surgery, Institute for Human Tissue Restoration, Severance Hospital, Yonsei University College of \\ Medicine, Seoul, Korea
}

\begin{abstract}
Background Classical flaps for perinasal defect reconstruction, such as forehead or nasolabial flaps, have some disadvantages involving limitations of the arc of rotation and two stages of surgery. However, a perforator-based flap is more versatile and allows freedom in flap design. We introduced our experience with reconstruction using a facial artery perforator-based propeller flap on the perinasal area. We describe the surgical differences between different defect subtypes.

Methods Between December 2005 and August 2013, 10 patients underwent perinasal reconstruction in which a facial artery perforator-based flap was used. We divided the perinasal defects into types $A$ and $B$, according to location. The operative results, including flap size, arc of rotation, complications, and characteristics of the perforator were evaluated by retrospective chart review and photographic evaluation.

Results Eight patients were male and 2 patients were female. Their mean age was 61 years (range, 35-75 years). The size of the flap ranged from $1 \mathrm{~cm} \times 1.5 \mathrm{~cm}$ to $3 \mathrm{~cm} \times 6 \mathrm{~cm}$. Eight patients healed uneventfully, but 2 patients presented with mild flap congestion. However, these 2 patients healed by conservative management without any additional surgery. All of the flaps survived completely with aesthetically pleasing results.

Conclusions The facial artery perforator-based flap allowed for versatile customized flaps, and the donor site scar was concealed using the natural nasolabial fold.
\end{abstract}

Keywords Perforator flap / Nose / Surgical flaps
Correspondence: Won Jai Lee Department of Plastic and Reconstructive Surgery, Institute for Human Tissue Restoration, Severance Hospital, Yonsei University College of Medicine, 50 Yonsei-ro, Seodaemungu, Seoul 120-749, Korea

Tel: +82-2-2228-2219

Fax: +82-2-393-6947

E-mail: pswjlee@yuhs.ac

Received: 27 May 2013 • Revised: 15 Jul 2013 - Accepted: 26 Jul 2013

pISSN: 2234-6163 • elSSN: 2234-6171 • http://dx.doi.org/10.5999/aps.2013.40.6.754 • Arch Plast Surg 2013;40:754-760

This article was presented as a poster at the Research and Reconstructive Forum May 12-13, 2011 in Daejeon, Korea.

No potential conflict of interest relevant to this article was reported.

\section{INTRODUCTION}

The perinasal area is a cosmetically and functionally important aesthetic subunit of the face because this region is located in the middle of the face and has considerable significance in appearance and expression. However, its successful and aesthetic reconstruction remains a challenge for plastic surgeons.

There are many surgical methods by which perinasal defects are reconstructed, including the free flap [1], forehead flap [2], nasolabial flap [3], and various local flaps [4,5]. In the case of a non-excessive defect, local flaps are preferred not only because they use less time-consuming procedures than free flaps but 
also because they offer optimal skin color, contour, and texture matching. Also, the nasolabial skin is the ideal donor site with regard to scar concealment in the natural nasolabial fold. Therefore, traditional local flaps such as nasolabial flaps are good flaps to use for perinasal defect reconstruction. However, the only drawback is that they require a two-stage reconstruction to achieve an aesthetically pleasing alar-cheek groove [3].

Recently, perforator flaps have become popular in many areas of reconstructive surgery. They provide design freedom and reduce donor-site morbidity. Many traditional local flaps including nasolabial flaps are based on the use of the axial facial artery with multiple perforators. Flaps based on one perforator of the facial artery have been introduced and are used more frequently because of their advantages over perforator flaps [6-12].

The facial artery perforator-based flap is the first true perforator flap in the face. It allows one-stage reconstruction and allows freedom in flap design. We reconstructed perinasal defects with island flaps based on a single perforator of the facial artery itself or its branches. In this article, we have presented our operative results, including surgical techniques and complications. Furthermore, we divided the defect into location subtypes and have explained both the surgical technique and characteristics of the perforators of each subtype.

\section{METHODS}

Between December 2005 and August 2013, a facial artery perforator-based flap procedure was performed on 10 patients at our institution. The operative results, including the cause of the defect, flap size, location of the defect, arc of rotation, and complications, were evaluated by a retrospective chart review and photographic evaluation. Furthermore, according to location, we divided the defects into types A and B. Type A is located in the sidewall of the nose, including the alar rim, and type $\mathrm{B}$ is located in the nostril floor, including the alar base. We compared the location and characteristics of the type A and B perforators.

\section{Surgical technique}

After tumor ablation or nostril stenosis surgery in our cases, the facial artery perforator along the nasolabial fold was found and marked with a handheld Doppler probe. A perforator near the defect was selected to accomplish a sufficient arc of rotation without tension. The flap was designed to cover the defect based on one perforator and to conceal the donor site in the nasolabial fold. An elliptical-shaped flap was designed for primary closure of the donor site. An incision on one side of the flap was made to the subcutaneous tissue. Undermining of the flap was performed until the perforators were identified. After the perfora- tors were identified, the flap was redesigned and an incision was made circumferentially.

Dissection of the pedicle was performed until tension-free transposition was achieved to cover the defect, and the distal perforators were sacrificed to ease flap movement. For protection against shear forces, we left a small cuff of subcutaneous fat around the perforator. After elevation of the flap, it was rotated to cover the defect without advancement, using the propeller flap concept, which is used for reconstruction of the lower extremity. In two cases, a full-thickness skin graft (FTSG) was applied to cover the remainder of the defect due to its large size. The donor site was closed using primary repair and concealed within the nasolabial fold.

In type A defect reconstruction, $180^{\circ}$ rotation propeller flaps were used. We performed more skeletonization than in type $\mathrm{B}$ due to a large arc of rotation. Therefore, a more careful perforator dissection was needed to ensure the safety of the flap. We needed thinner flaps in this reconstruction compared to type $B$. We therefore performed a defatting step after flap elevation. In type $\mathrm{B}$ defect reconstruction, $120^{\circ}$ rotation propeller flaps were used. Because of the deep nature of this defect, we needed thicker flaps than in type $A$ and thus there was no need for a defatting step.

\section{RESULTS}

The patients' age range was 35 to 75 years (mean, 61 years). Eight patients were male and 2 patients were female. Five patients were smokers. Seven patients had been diagnosed with skin cancer (basal cell carcinoma [BCC], squamous cell carcinoma [SCC], or malignant melanoma), and one patient had metastatic adenocarcinoma of the liver. One patient had a venous malformation, and the remaining patients had nostril stenosis, whose defect was the result of nostril stenosis release (Table 1). All of the operations were performed under general anesthesia. In two cases, FTSGs were needed to cover the defects, which could not be reconstructed using only a facial artery perforatorbased flap because of their large sizes. The patient who had nostril stenosis bilaterally had surgery using a bilateral facial artery perforator-based flap. Patient follow-up time ranged between 3 and 6 months (average, $4.8 \pm 1.5$ months).

In all cases, we identified a suitable perforator of the facial artery or its branches with which to reconstruct the perinasal defect. All flaps were based on a single arterial perforator. The skin overlying the flap pedicle and the donor site could be closed with primary repair without tension and concealed within the nasolabial fold. The flap and skin around the defect were well matched with regard to both color and texture. 
Table 1. Demographic data and reconstruction detail of patients

\begin{tabular}{|c|c|c|c|c|c|c|c|c|}
\hline Patient No. & $\begin{array}{c}\text { Sex/Age } \\
\text { (yr) }\end{array}$ & Cause & Location & Type & Flap size $(\mathrm{cm})$ & Operative methods & Perforators & Complications \\
\hline 1 & $\mathrm{M} / 48$ & Venous malformation & Nasal sidewall & A & $3 \times 6$ & $180^{\circ}$ rotation+FTSG & LNAP & Temporary venous congestion \\
\hline 2 & $\mathrm{M} / 72$ & $\mathrm{BCC}$ & Nasal sidewall & A & $3 \times 5$ & $180^{\circ}$ rotation+FTSG & LNAP & Temporary venous congestion \\
\hline 3 & M/35 & SCC & Alar base, nasal floor & B & $2 \times 3$ & $120^{\circ}$ rotation & SLAP & None \\
\hline 4 & $\mathrm{M} / 71$ & SCC & Columella, nasal floor, bilateral & B & $3 \times 4$ & $120^{\circ}$ rotation & FAP & None \\
\hline 5 & $\mathrm{M} / 64$ & Nostril stenosis & Vestibule, bilateral & B & $1 \times 1.5$ & $120^{\circ}$ rotation & SLAP & None \\
\hline 6 & $\mathrm{M} / 75$ & $\mathrm{BCC}$ & Nasal ala & A & $3 \times 3.5$ & $180^{\circ}$ rotation & FAP & None \\
\hline 7 & $\mathrm{M} / 63$ & SCC & Nasal floor & B & $2 \times 5$ & $120^{\circ}$ rotation & SLAP & None \\
\hline 8 & $\mathrm{~F} / 69$ & Malignant melanoma & Nasal ala & A & $3 \times 4$ & $180^{\circ}$ rotation & FAP & None \\
\hline 9 & $\mathrm{~F} / 61$ & $\mathrm{BCC}$ & Nasal ala & A & $3 \times 4$ & $180^{\circ}$ rotation & FAP & None \\
\hline 10 & $\mathrm{M} / 53$ & Metastatic adenocarcinoma & Nasal ala & A & $2 \times 4$ & $180^{\circ}$ rotation & FAP & None \\
\hline
\end{tabular}

\section{Fig. 1. Case 1 (type A defect reconstruction)}

A 48-year-old man with venous malformation (patient number 1). (A) A preoperative photograph. (B) Designed flap after the resection of the venous malformation ( $\star$, flap coverage area; $\$$, full thickness skin graft coverage area). (C) Late postoperative result 6 months after the procedure. He was satisfied with the aesthetic result.
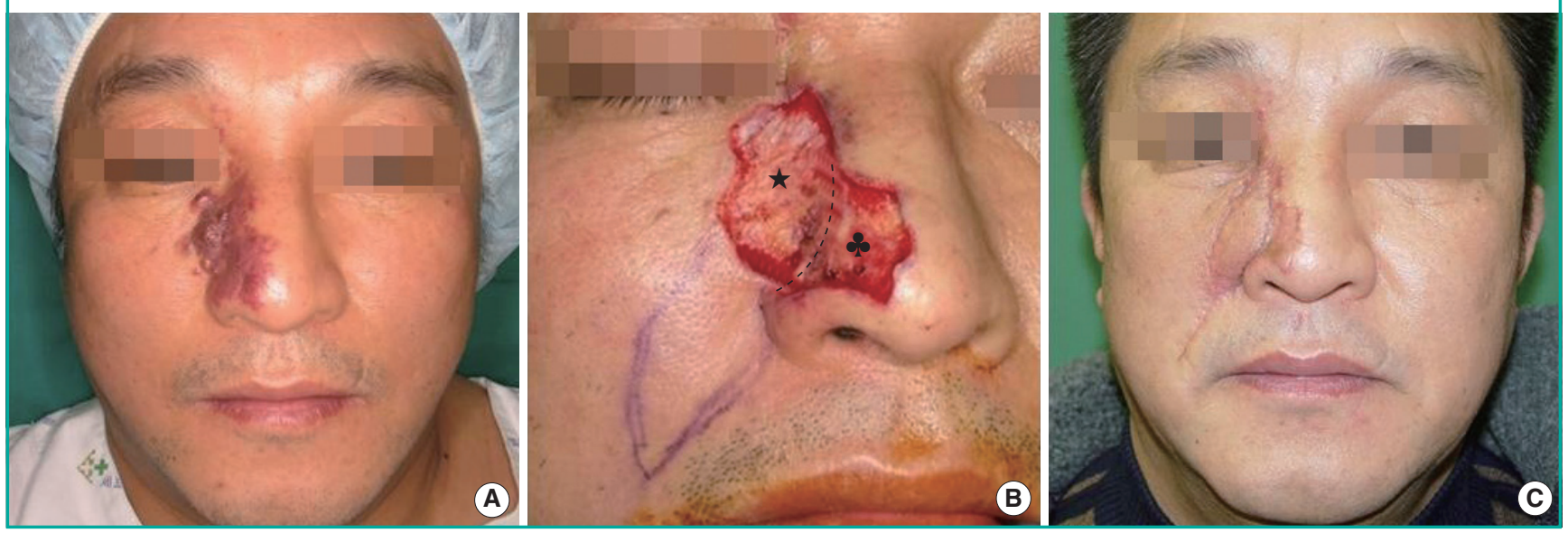

In the type A defect reconstruction cases, the flaps were based on perforators of the facial artery and lateral nasal artery. The defects were covered with a $180^{\circ}$ rotation. In two cases, mild venous congestion was seen on postoperative day 1 but healed with conservative management. Such congestion was assumed to be due to the large arc of rotation and large defect size. In the type B defect reconstruction cases, the flaps were based on perforators of the facial artery and superior labial artery. The defects were covered with a $120^{\circ}$ rotation. All of the flaps survived. Good cosmetic and functional results of the facial artery perforator-based flap were achieved and did not require any revisions on follow-up. All of the patients were satisfied with the results.

\section{Case 1 (type Adefect reconstruction)}

A 48-year-old man underwent excision of a venous malformation in the right nasal sidewall. After the defect was detected, we decided to cover it with a facial artery perforator-based flap that was
$3 \mathrm{~cm}$ in width and $6 \mathrm{~cm}$ in length. A perforator from the lateral nasal artery was identified. A FTSG was needed for an additional procedure on the caudal area of the defect because of the large size of the defect. Mild venous congestion was seen on postoperative day 1 but healed with conservative management (Fig. 1).

\section{Case 2 (type Adefect reconstruction)}

A 72-year-old man underwent BCC excision in the right nasal sidewall. Following cancer resection, he had a large defect over the nasal sidewall. A perforator from the lateral nasal artery was used. A $180^{\circ}$ rotation island flap that was $3 \mathrm{~cm}$ in width and 5 $\mathrm{cm}$ in length was designed, and a FTSG was needed for an additional procedure on the nasal dorsum. Mild flap congestion on postoperative day 1 was resolved without surgery (Fig. 2).

\section{Case 3 (type $B$ defect reconstruction)}

A 35-year-old man had a SCC on the right alar base and nasal 


\section{Fig. 2. Case 2 (type A defect reconstruction)}

A 72-year-old man with BCC (patient number 2). (A) A preoperative photograph. (B) Designed flap after the resection of the BCC. (C) Intraoperative photography of a perforator. (D) Late postoperative result 3 months after the procedure. BCC, basal cell carcinoma.
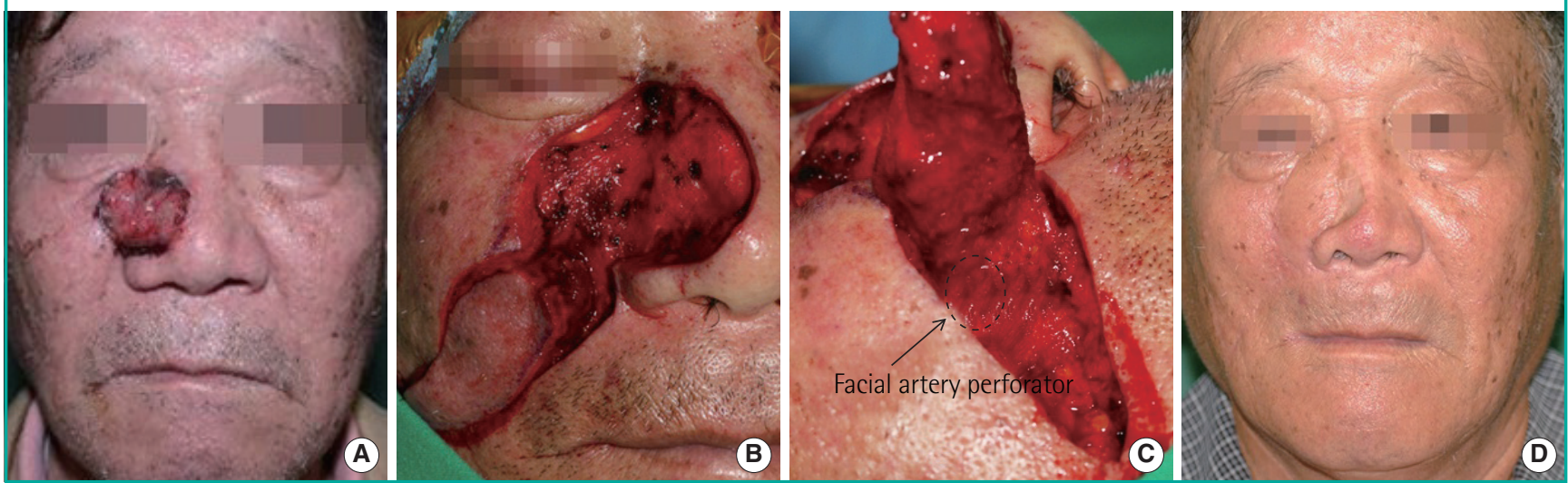

\section{Fig. 3. Case 3 (type B defect reconstruction)}

A 35-year-old man with squamous cell carcinoma (patient number 3). (A) Intraoperative photography of perforator. (B) Immediate postoperative result. (C) Late postoperative result 6 months after the procedure.
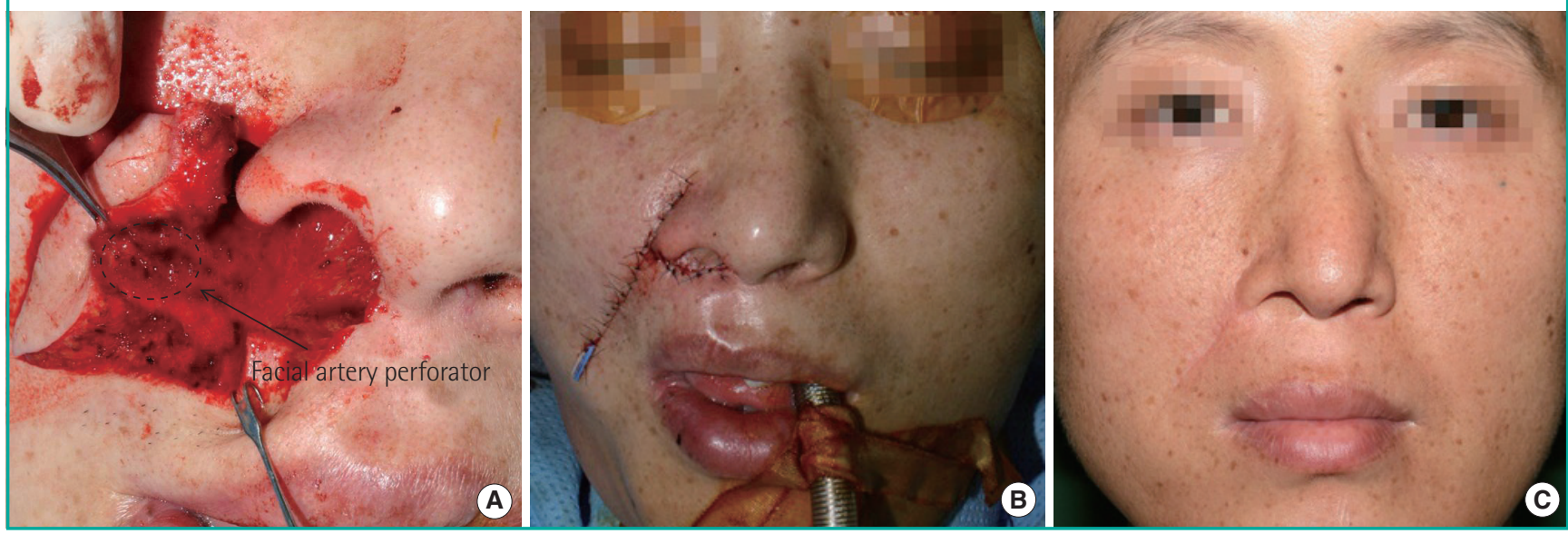

floor. Following cancer resection, a perforator of the superior labial artery was identified at the nasolabial fold using a handheld Doppler probe. Dissection of the pedicle was performed until tension-free rotation was achieved to cover the defect. A $120^{\circ}$ rotation island flap that was $2 \mathrm{~cm}$ in width and $3 \mathrm{~cm}$ in length was elevated and pivoted on this perforator. A 3-dimensional reconstruction of the deep defect was achieved using the versatile perforator flap (Fig. 3).

\section{Case 4 (type $B$ defect reconstruction)}

A 71-year-old man underwent excision of SCC on the columella and part of the septal cartilage. After the defect was localized, we decided to cover the deep nasal floor defect and reconstruct the columella with a bilateral facial artery perforator-based flap that was $3 \mathrm{~cm}$ in width and $4 \mathrm{~cm}$ in length. We customized the reconstruction using a facial artery perforator-based flap (Fig. 4).

\section{DISCUSSION}

The perinasal regions are anatomic facial subunits that are important with regard to functional and aesthetic characteristics. Thus, the goal of perinasal defect reconstruction includes both functional and aesthetic restoration. Although many methods, including free flaps, have been used, regional flaps are preferred for reconstruction of perinasal defects. Regional flaps will always provide a superior aesthetic result because these tissues have the same color and texture as the defect [8].

Regional flaps such as traditional nasolabial flaps, island nasolabial flaps, and V-Y advancement flaps are considered the first choices for reconstruction of perinasal defects. These flaps are also supplied by perforators from the facial artery $[8,13]$. However, some of the defects are negligibly reconstructed by such conventional flaps. Freestyle flaps based on a single perforator 
Fig. 4. Case 4 (type B defect reconstruction)

A 71-year-old man with squamous cell carcinoma on a columella (patient number 4). (A) A preoperative photograph. (B) After a resection of the cancer, a large defect of columella, nasal septum, vestibule, and floor was created. (C) Immediate postoperative result after the procedure of $180^{\circ}$ rotation with a facial artery perforator island flap on both side. (D) Late postoperative result 6 months after the procedure. He was satisfied with the aesthetic result.
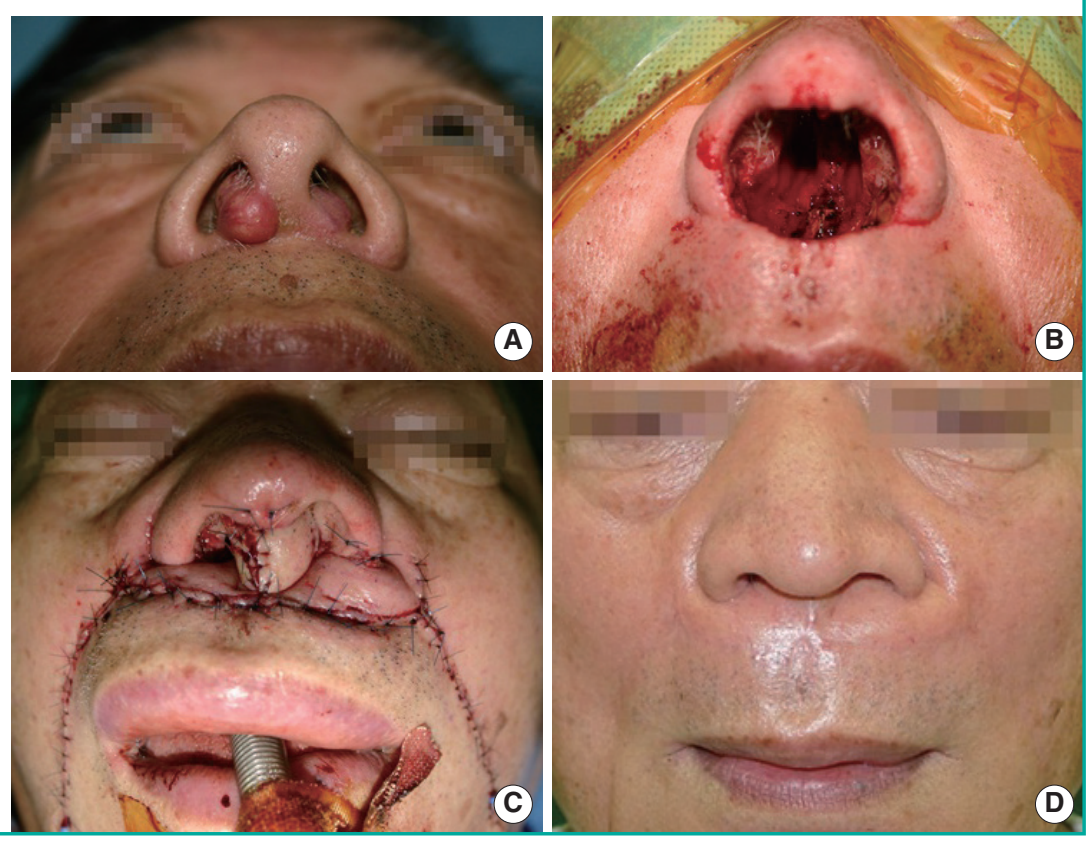

can allow for customized reconstruction of perinasal defects $[8,9,11]$.

Since the term perforator flap was first used by Koshima and Soeda [14] in 1989, numerous perforator flaps have been designed, which allow thinner flaps to be tailored for more accurate reconstruction. Whether it is correct to call this nasolabial island flap a perforator flap is a subject of debate. According to the Gent Consensus Conference [15], a perforator should pierce the deep fascia before reaching the skin. Because there is no deep fascia layer in the face and the vessels pierce the superficial muscular aponeurotic system layer before reaching the skin, this flap can be also called a perforator flap [9].

We used an island nasolabial flap-based facial artery perforator and the facial artery branches' perforator. The term "perforatorbased" is a flap that is truly based on the perforator itself without sacrificing the proximal vessel [16]. Therefore, the facial artery and arteries within its subsystem are saved and not dissected. We used methods involving a propeller flap in all of our cases. The term "propeller flap" is an island flap that reaches the recipient site by axial rotation [17]. This method has been a useful reconstructive tool in various areas, especially the lower leg, and can achieve good cosmetic and functional results. With this method, we were able to accomplish good aesthetic results.

The facial artery perforator-based flap is supported by the study of angiosomes of the facial region, which showed that the facial arterial system supplies the cutaneous areas from the submental region to the medial two-thirds of the face [18]. Although Nakajima et al. [19] reported variations in the facial artery and its branches, the superior labial, lateral nasal, and angular arteries are consistent in $98 \%$ of cases, with $2 \%$ of facial arteries terminating at the alar base level [20]. More detailed cadaveric studies have presented a rich perforator density in the perioral and perinasal areas [21]. In another cadaveric study, it was shown that an average of five perforators exist per facial artery with a mean diameter of $0.96 \mathrm{~mm}$. The perforators were selectively injected with diluted ink solution, and the mean size of all of the injected skin areas was $8.05 \mathrm{~cm}^{2}$. Seven main, reliable types of perforator territories were identified: the posterior area of the horizontal ramus of the mandible, the anterior area of the horizontal ramus of the mandible, the inferior labial area, the commissural area, the jugal area, the nasolabial area, and the subpalpebral area. Defining these territories will help in the proper design of a flap, and various nasolabial flaps have been designed based on the rich perforasomes of the perioral and perinasal areas [22].

We classified the defects according to their location. Type A cases were located in the sidewall of the nose, including the alar rim, and type B cases were located in the nostril floor or columella, including the alar base. The reconstruction was quite different in types A and B. In type A defect reconstruction, $180^{\circ}$ rotation propeller flaps were used. Therefore, more careful perforator dissection was needed due to the large arc of rotation. Considering the aesthetic results and to prevent postoperative "pin-cushioning," we needed a thinner flap for this reconstruction. Therefore, we performed a defatting step after elevating the flap. We usually used a lateral nasal artery perforator, including 
the facial artery perforator. In type B defect reconstruction, $120^{\circ}$ rotation propeller flaps were used. Because this defect is usually located in a deeper area, we needed a thicker flap, obviating the need for a defatting step. Furthermore, we paid careful attention to the flap design due to the 3-dimensional reconstruction. We usually used the superior labial artery perforator, including the facial artery perforator.

It is hypothesized that venous drainage of the facial artery perforator flap is similar to that described for digital island flaps. This drainage is contained within the fibrofatty tissue around the artery [8]. However, other studies report that it is better not to leave a cuff of fat around the perforator as it may cause pedicle torsion or kinking. Furthermore, these studies state that careful dissection will always allow for identification of at least one vein, which will drain the skin flap [9]. We partially dissected the pedicle to transpose the flap, covering the defects. In such cases, we left a small cuff of fat around the perforator.

All patients had satisfying results and did not need any additional revisions. Therefore, as per our preoperative plan, perinasal reconstruction with facial artery perforator-based flaps was performed using a one-stage reconstruction without any complications. Using this approach, prior surgeries that involved two stages using nasolabial or forehead flaps can now be performed as a one-stage procedure.

This method can be applied to small to moderate-sized perinasal defects. For the primary repair of the donor site, flap sizes are limited. In our two cases, we needed FTSGs for an additional procedure due to the large size of the defect. In these cases, there were compromised aesthetic results. Another possible negative aspect of this method is the time required. Perforator dissection is a more difficult procedure than the use of other local flaps that do not require perforator dissection. However, with experience, the duration of such a dissection could decrease.

Using a facial artery perforator-based flap allowed greater freedom in rotation, mobility, and flap design for the reconstruction of perinasal defects. Other advantages are the possibility of a one-stage reconstruction and customization. In addition, the use of this flap increases aesthetic results, as it uses a local flap and conceals the donor scar within a natural fold. Therefore, we believe that a facial artery perforator-based flap may provide an alternative reconstruction method for peri nasal defects.

\section{REFERENCES}

1. Menick FJ, Salibian A. Microvascular repair of heminasal, subtotal, and total nasal defects with a folded radial forearm flap and a full-thickness forehead flap. Plast Reconstr Surg 2011;127:637-51.
2. Menick FJ. Nasal reconstruction with a forehead flap. Clin Plast Surg 2009;36:443-59.

3. Cameron RR, Latham WD, Dowling JA. Reconstructions of the nose and upper lip with nasolabial flaps. Plast Reconstr Surg 1973;52:145-50.

4. Chu EA, Byrne PJ. Local flaps I: bilobed, rhombic, and cervicofacial. Facial Plast Surg Clin North Am 2009;17:349-60.

5. Kwon KH, Lee DG, Koo SH, et al. Usefulness of v-y advancement flap for defects after skin tumor excision. Arch Plast Surg 2012;39:619-25.

6. Krijgh DD, Mureau MA. Aesthetic and functional outcome following perioral defect reconstruction using the facial artery perforator flap. J Reconstr Microsurg 2012;28:529-38.

7. Kim SW, Kim YH, Kim JT. Angular artery perforator-based transposition flap for the reconstruction of midface defect. Int J Dermatol 2012;51:1366-70.

8. Hofer SO, Posch NA, Smit X. The facial artery perforator flap for reconstruction of perioral defects. Plast Reconstr Surg 2005; 115:996-1003.

9. D’Arpa S, Cordova A, Pirrello R, et al. Free style facial artery perforator flap for one stage reconstruction of the nasal ala. $\mathrm{J}$ Plast Reconstr Aesthet Surg 2009;62:36-42.

10. Sohn WI, Choi JY, Seo BF, et al. Reconstruction of nasal ala with nasolabial perforator flap after cancer removal. Head Neck Oncol 2012;4:83.

11. Demirseren ME, Afandiyev K, Ceran C. Reconstruction of the perioral and perinasal defects with facial artery perforator flaps. J Plast Reconstr Aesthet Surg 2009;62:1616-20.

12. Karsidag S, Ozcan A, Sumer O, et al. Single-stage ala nasi reconstruction: lateral nasal artery perforator flap. J Craniofac Surg 2010;21:1887-9.

13. Feinendegen DL, Langer M, Gault D. A combined V-Y advancement-turnover flap for simultaneous perialar and alar reconstruction. Br J Plast Surg 2000;53:248-50.

14. Koshima I, Soeda S. Inferior epigastric artery skin flaps without rectus abdominis muscle. Br J Plast Surg 1989;42:645-8.

15. Blondeel PN, Van Landuyt KH, Monstrey SJ, et al. The "Gent" consensus on perforator flap terminology: preliminary definitions. Plast Reconstr Surg 2003;112:1378-83.

16. Kim JT. New nomenclature concept of perforator flap. Br J Plast Surg 2005;58:431-40.

17. Pignatti M, Ogawa R, Hallock GG, et al. The "Tokyo" consensus on propeller flaps. Plast Reconstr Surg 2011;127:716-22.

18. Houseman ND, Taylor GI, Pan WR. The angiosomes of the head and neck: anatomic study and clinical applications. Plast Reconstr Surg 2000;105:2287-313.

19. Nakajima H, Imanishi N, Aiso S. Facial artery in the upper lip and nose: anatomy and a clinical application. Plast Re- 
constr Surg 2002;109:855-61.

20. Niranjan NS. An anatomical study of the facial artery. Ann Plast Surg 1988;21:14-22.

21. Ng ZY, Fogg QA, Shoaib T. Where to find facial artery perforators: a reference point. J Plast Reconstr Aesthet Surg
2010;63:2046-51.

22. Qassemyar Q Havet E, Sinna R. Vascular basis of the facial artery perforator flap: analysis of 101 perforator territories. Plast Reconstr Surg 2012;129:421-9. 